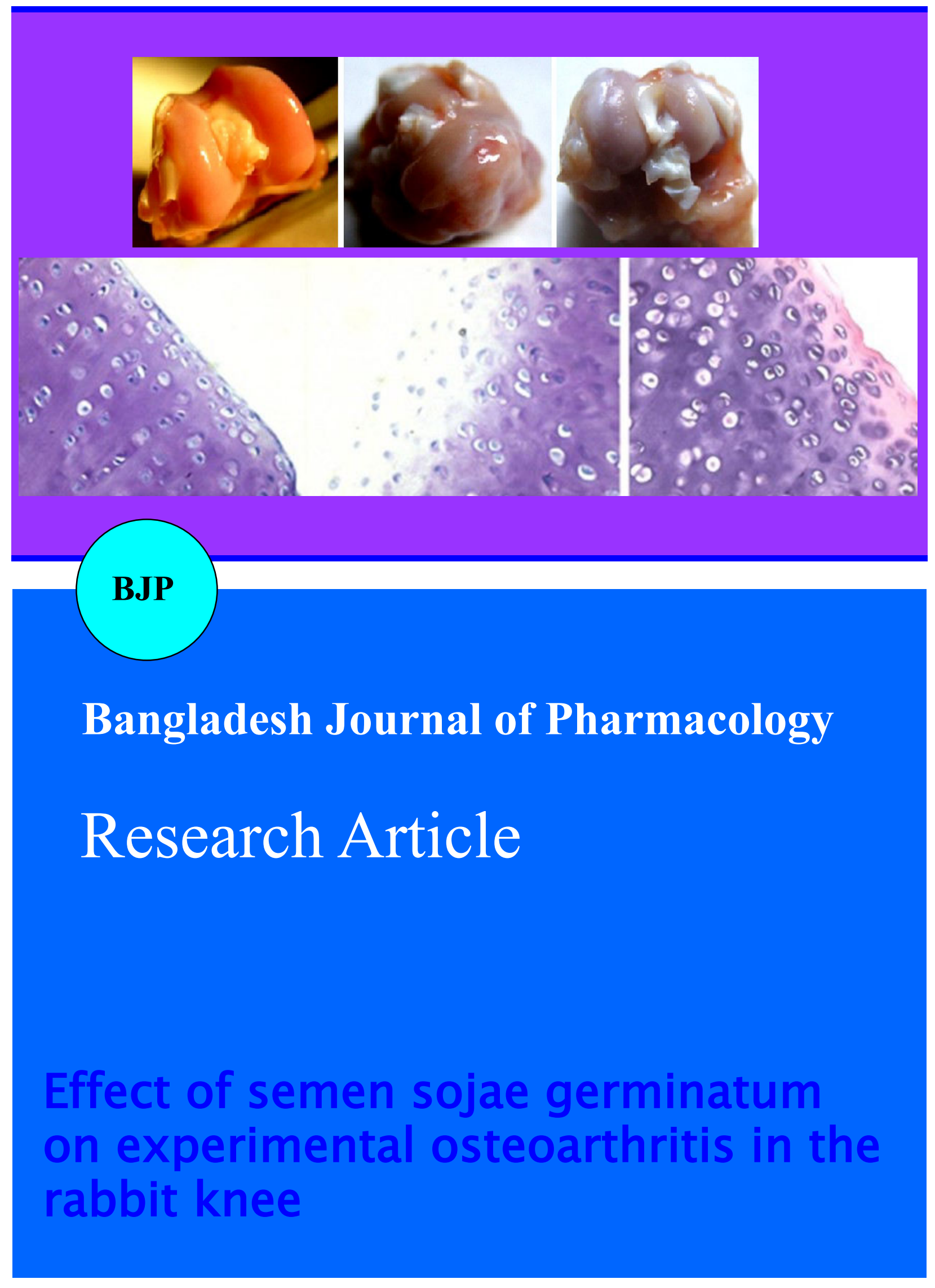




\title{
Effect of semen sojae germinatum on experimental osteoarthritis in the rabbit knee
}

\author{
Bingshu He1 and Jun Wang ${ }^{2}$ \\ ${ }^{1}$ Department of Orthopedic Surgery, Hubei Woman and Child Hospital, Wuhan 430070, China; ${ }^{2}$ Department of \\ Pharmacy, Medical College, Wuhan University of Science and Technology, Wuhan 430065, China.
}

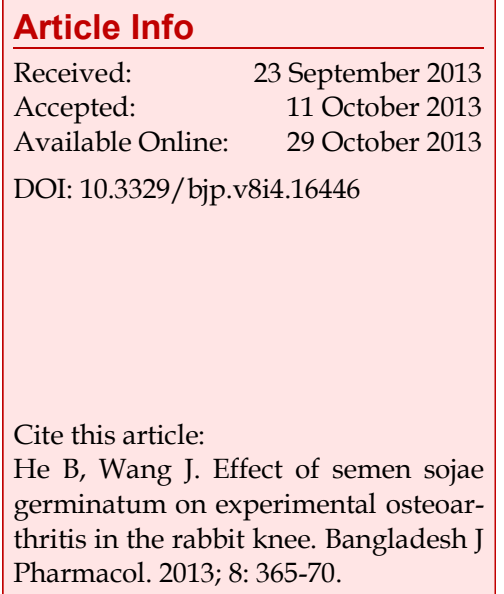

\begin{abstract}
As a complementary therapeutic approach, soy ingredients, such as soy protein and soybean unsaponifiables, recently attract more attention for relieving symptoms of osteoarthritis (OA). Semen sojae germinatum (SSG) processed from bean-sprout coming from germinating Glycine max L. Merr. is a traditional Chinese herbal medicine treating knee OA. We evaluated the effect of SSG as feed on the development of anterior cruciate ligament transaction (ACLT) -induced knee OA in rabbits. The results showed that SSG reduced the severity of cartilage degeneration in macroscopic grading scale, and delayed the degradation of the cartilage matrix. The contents of prostaglandin $\mathrm{E}_{2}\left(\mathrm{PGE}_{2}\right)$, nitric oxide $(\mathrm{NO})$, tumor necrosis factor $(\mathrm{TNF})-\mathrm{a}$, interleukin-6 (IL-6) and IL-1 $\beta$ in synovial fluid, mRNA and protein expressions of matrix metalloproteinases (MMP)-3, MMP-13 in cartilage were effectively decreased in SSG group. These results demonstrate that SSG had a protective effect against the development of knee OA.
\end{abstract}

\section{Introduction}

Osteoarthritis (OA) characterized by progressive degeneration of the articular cartilage and accompanied by subchondral bone sclerosis and synovial inflammation is a degenerative joint disease afflicting an increasingly older population (Felson et al., 2000). Approximately $10 \%$ of adults aged 60 years or older suffer from OA of the knee (Scott and Kowalczyk, 2008). There is still no curative treatment for this disease despite availability of a large number of therapeutic options including nonpharmacological, pharmacological, and surgical therapies. Drug therapy includes non-opioid analgesics such as non-steroidal antiinflammatory drugs (NSAIDs), topical analgesics, opioid analgesics and intra-articular steroid injection. Such treatments can only relieve some symptoms of OA, and may prove ineffective in some patients (Tramèr et al., 2000). The most common therapy for treating OA is NSAIDs, but their use may be restricted by adverse gastrointestinal effects including serious occurrences of bleeding causing many deaths (Felson et al., 2000). Hence, there appears to be a need for drugs with good efficacy and low toxicity in the treatment of OA.

Chinese medicine, such as herbal medicinal products, has been proved to be effective in the treatment of OA through slowing down cartilage degeneration, promoting articular cartilage repair and inhibiting synovial inflammation (Wang et al., 2010a). Importantly, no serious side effects were reported with any herbal intervention (Cameron et al., 2009). In traditional Chinese medicine, the condition which is congruent with OA is called "Bi syndrome" (painful obstruction syndrome), and more specifically, "Bi syndrome of bone". "Bi syndrome" manifests as pain, soreness, or numbness of muscles, tendons and joints, and is the result of the body being "invaded" by the external climatological factors of Wind, Cold, Heat, and/or Dampness (Hua and O'Brien, 2010). Semen sojae germinatum (SSG) is one of Chinese folk recipes treating "Bi syndrome of bone" recorded by "Shen 
Nong's Herbal Classic" and "Compendium of Materia Medica". Indeed, SSG is processed bean sprout coming from germinating Chinese black soybean (Glycine max L. Merr.) variety, and has been included in Edition 2010 Pharmacopoeia of People's Republic of China as a new kind. Although SSG has been widely used in traditional Chinese medicine clinical practices based on the ancient Chinese perception and experience, nowadays, study on the modern pharmacological effects of SSG is still rare.

In this study, we attempted to determine whether SSG has the protective effect on articular cartilage in a rabbit knee OA model induced by anterior cruciate ligament transaction (ACLT), and study its mechanism.

\section{Materials and Methods}

\section{SSG preparation}

SSG was prepared referring to Edition 2010 Pharmacopoeia of People's Republic of China. Chinese black soybeans (Glycine max L. Merr.) were sprouted. When the sprouts are $1 \mathrm{~cm}$ high, spread them out, let wind blow them to a half dry, then dry them in the sun to prepare SSG. Subsequently, Lophatherum gracile and Juncus effusus (Lophatherum gracile $2 \mathrm{~kg}$, Juncus effusus $1 \mathrm{~kg} /$ SSG $100 \mathrm{~kg}$ ) were put in the pot, and decocted twice (first time $60 \mathrm{~min}$, second time $30 \mathrm{~min}$ ). Then the decoction solution was mixed and filtrated. The filtrate and SSG were both put in a pot, then cooked until the filtrate was all absorbed by SSG. The total amount of daidzin $\left(\mathrm{C}_{21} \mathrm{H}_{20} \mathrm{O}_{9}\right)$ and genistein $\left(\mathrm{C}_{21} \mathrm{H}_{20} \mathrm{O}_{10}\right)$ accounted for no less than $0.08 \%(\mathrm{w} / \mathrm{w})$ of the dry material using HPLC method as described in Edition 2010 Pharmacopoeia of People's Republic of China. Finally, the prepared SSG was dried and smashed to make the standard granulated feed.

\section{Animal and experimental arthritis}

New Zealand white rabbits $(2.0-2.5 \mathrm{~kg})$ were obtained from the Laboratory Animal Center of Wuhan University (Wuhan, Hubei, China). Animal care and treatment were in accordance with the guidelines of the Laboratory Animal Management and Review Committee of Wuhan University of science and technology. Rabbits were randomly divided into 3 groups of 5 rabbits each: sham operated group, the OA model control group and SSG group. Rabbits in SSG group were fed with the diet made of prepared SSG, while rabbits in sham operated group and the OA model control group were fed with normal diet. The basis of the food which was used to prepare the SSG containing food was the same as the food given to the control animals

After the animals were fed with the corresponding diet for 4 weeks, OA was induced by ACLT and excisions of medial menisci in the right knees (Yoshioka et al., 1996) on the rabbits of OA model control group and SSG group. The sham operated group only had the right joint cavity opened and then the incision was sutured. The corresponding diet was given unceasingly.

\section{Gross pathology observation and histological evalua- tion}

After 8 weeks, the rabbits were sacrificed and femoral condyles were collected for observation. The depth of erosion was graded on a scale of 0-4 (Pelletier et al., 1998). All samples were graded by three independent observers unaware of the treatment groups. The cartilage samples were embedded in paraffin, and standard frontal sections of $5 \mu \mathrm{m}$ sections were prepared and stained with toluidine blue in the lateral part of the femoral condyle cartilage according to gross observation (Cake et al., 2000).

Prostaglandin (PG)E2, nitric oxide (no), tumor necrosis factor (tnf)-a, interleukin (il)-6 and il-1ßin synovial fluid

After the rabbits were sacrificed, knees were washed with $1 \mathrm{~mL}$ of $0.9 \%(\mathrm{w} / \mathrm{v})$ physiological saline solution through the patellar tendon. The contents of $\mathrm{PGE}_{2}$ and $\mathrm{NO}$ in synovial fluid were measured by the correspondding kits (Chinese Academy of Medical Sciences, Beijing, and Jiancheng Co., Nanjing, China, respectively). Concentrations of TNF-a, IL-6 and IL-1 $\beta$ in synovial fluid were measured using ELISA kits (Jiancheng Co., Nanjing, China).

mRNA and protein expression of matrix metalloproteinases ( $m m p)-3$ and mmp-13 in cartilage

The expression levels of MMP-3 and MMP-13 mRNA in

\section{Table I}

Primer sequences used in the study

\begin{tabular}{|c|c|c|}
\hline Gene & Length & Sequence of primers \\
\hline \multirow[t]{2}{*}{ MMP-3 } & \multirow[t]{2}{*}{72 bp } & Sense 5'-AGCCAATGGAAATGAAAACTCTTC-3' \\
\hline & & Antisense 5'-CCAGTGGATAGGCTGAGCAAA-3' \\
\hline \multirow[t]{2}{*}{ MMP-13 } & \multirow[t]{2}{*}{$60 \mathrm{bp}$} & Sense 5' - TTT TGA AGA CAC GGG CAA G -3' \\
\hline & & Antisense 5'- TCA TCA TAG CTC CAG ACT TGG TT -3' \\
\hline \multirow[t]{2}{*}{ GAPDH } & \multirow[t]{2}{*}{$66 \mathrm{bp}$} & Sense 5'- AGC CAC ATC GCT CAG ACA -3' \\
\hline & & Antisense 5'- GCC CAA TAC GAC CAA ATC C -3' \\
\hline
\end{tabular}




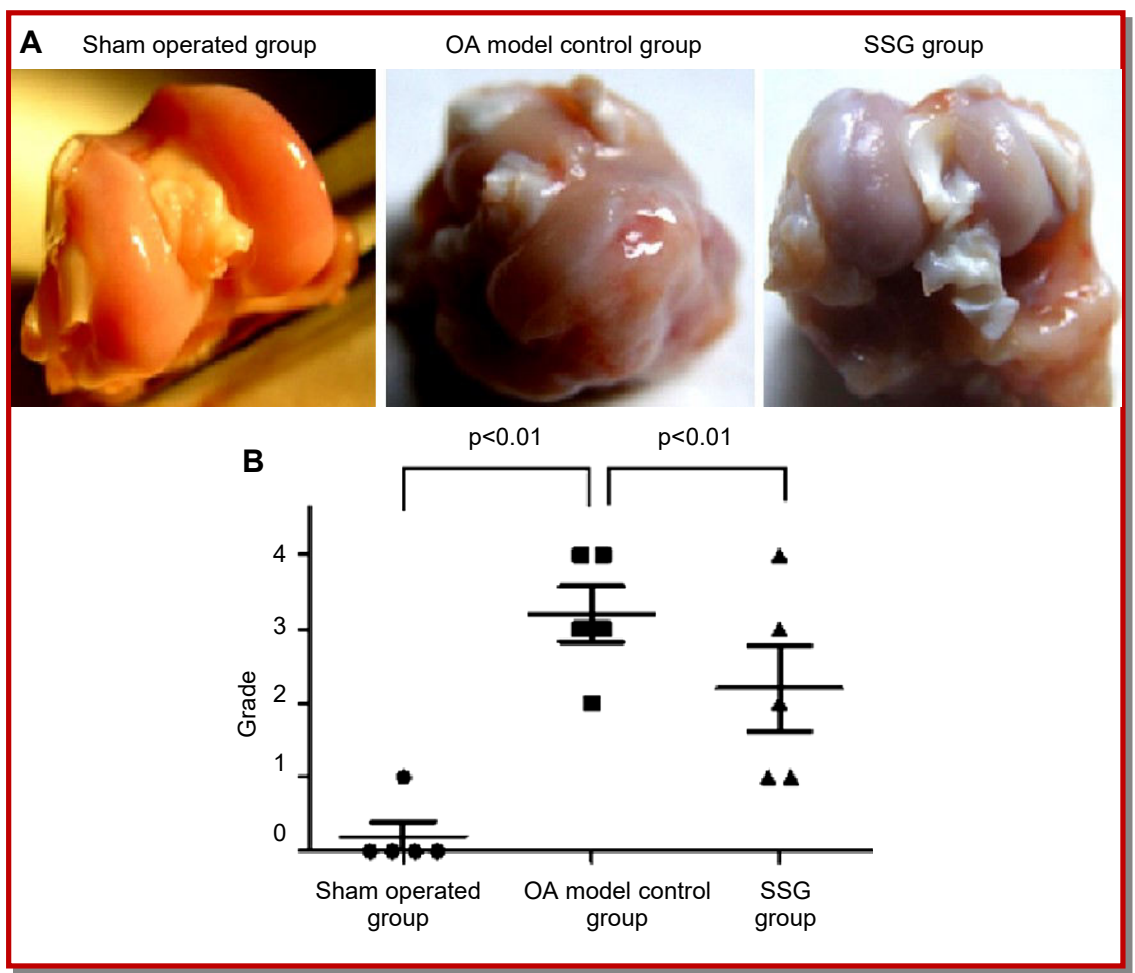

Figure 1. Gross pathological observation of the femoral condyle. A: Representative images of the femoral condyle from rabbits in sham operated, OA model control and SSG groups; B: Macroscopic grading scale of cartilage.

cartilage were detected by quantitative real-time PCR using TaqMan probes 59-fluorescent labeled with either FAM or VIC in a thermal cycler (ABI PRISM 7500 Sequence Detector System, Applied Biosystems, Foster City, CA, USA), employing glyceraldehyde-3phosphate dehydrogenase (GAPDH) gene as a reference gene. The sequences of each primer set (Invitrogen, Carlsbad, CA) used are given in Table I.

The protein expression levels of MMP-3 and MMP-13 were detected by Western blot. Proteins in cartilage were separated by SDS-PAGE and proteins were transferred onto a polyvinylidene difluoride membrane by electroblotting. Membrane was blocked overnight and then incubated for 2 hours with a 1:1000 dilution of goat polyclonal MMP-3 or rabbit polyclonal MMP-13 antibody (Santa Cruz, CA). After incubation with the secondary antibody, proteins were detected with an ECL chemiluminescence detection kit (Advansta, USA.) and scanned. The amount of protein expression was corrected by the amount of GAPDH in the same sample.

\section{Data analysis}

Comparisons between groups were per-formed with one-way ANOVA analysis using SPSS 11.5 software. The data were reported as means \pm standard deviation (SD). Statistical significance was defined by the value of $\mathrm{p}<0.05$.

\section{Results}

Knee articular cartilage of rabbits in sham operated group is smooth and lustrous. In the OA model control group, the articular cartilage surface was rough and had some superficial leakage and ulcers. In SSG group, cartilage lesions associated with OA were clearly seen, but the severity was milder than in OA group (Figure 1 A). Macroscopic grades in SSG group were significantly lower than those in the OA model control group $(\mathrm{p}<$ 0.05 ) as seen in Figure 1B, suggesting SSG can reduce the severity of the cartilage degeneration in macroscopic grading scale.

Articular cartilage consists of chondrocytes sparsely embedded within an abundant extracellular matrix which is composed essentially of water, proteoglycans, collagens and non-collagenous proteins (Cake et al., 2000). And cartilage proteoglycan loss is believed as one of the hallmarks of OA, because proteoglycans have the properties of resisting compression and generating the swelling pressure due to their affinity for water (Echtermeyer et al., 2009). Toluidine blue staining for proteoglycans (Getzy et al., 1982) showed a distinct reduction of these compounds in the cartilage matrix of OA rabbits. In SSG group, the matrix degradation was less evident than OA group (Figure 2).

Pro-inflammatory mediators alter matrix homeostasis and participate in the destruction of articular cartilage, thereby contributing to OA. It has been shown that OA 


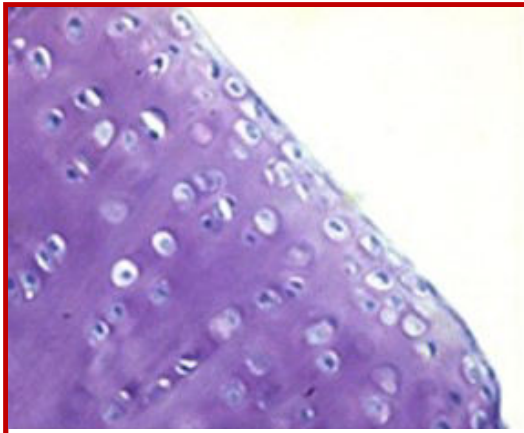

A

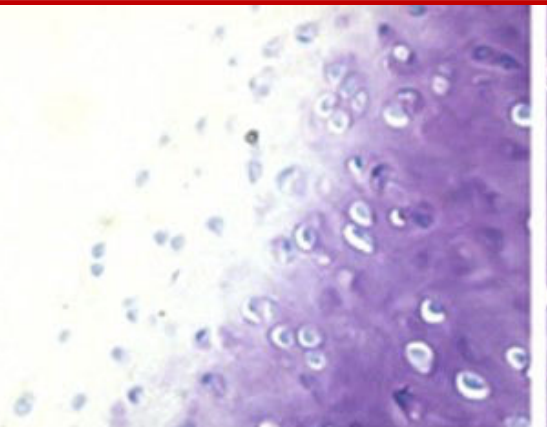

B

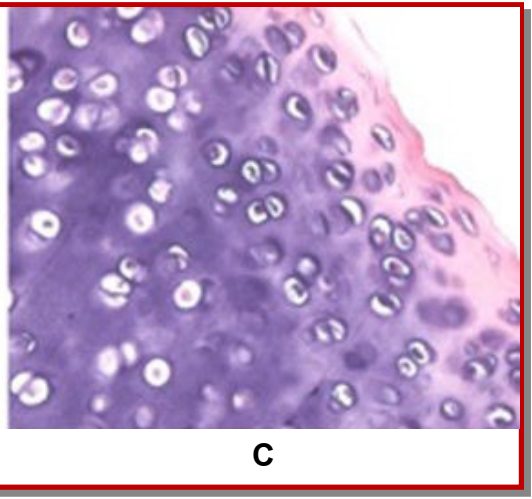

C

Figure 2. Histologic examination of cartilage change in rabbits of sham operated (A), OA model control (B) and SSG (C) groups. Cartilages were stained with toluidine blue. (original magnification $\times 100$ )

Table II

Concentration of PGE2, NO, TNF- $\alpha$, IL-6 and IL-1ßin synovial fluids from knees of rabbit OA

\begin{tabular}{|lccccc|} 
Group & PGE2 $/ \mathrm{ng} / \mathrm{mL}$ & $\mathrm{NO} / \mu \mathrm{mol} / \mathrm{L}$ & $\mathrm{TNF}-\mathrm{a} / \mathrm{ng} / \mathrm{mL}$ & $\mathrm{IL}-1 \beta / \mathrm{pg} / \mathrm{mL}$ & $\mathrm{IL}-6 / \mathrm{pg} / \mathrm{mL}$ \\
\hline Sham operated & $2.2 \pm 0.4$ & $29.8 \pm 8.2$ & $0.002 \pm 0.0$ & $8.5 \pm 0.9$ & $0.1 \pm 0.03$ \\
OA model control & $3.1 \pm 0.3^{\mathrm{a}}$ & $151.1 \pm 21.2^{\mathrm{a}}$ & $15.1 \pm 3.1^{\mathrm{a}}$ & $322.2^{\mathrm{a}} \pm 84.5^{\mathrm{a}}$ & $219.7 \pm 45.5^{\mathrm{a}}$ \\
SSG & $2.7 \pm 0.4^{\mathrm{b}}$ & $122.6 \pm 26.2^{\mathrm{b}}$ & $11.7 \pm 2.6^{\mathrm{b}}$ & $198.2 \pm 74.5^{\mathrm{c}}$ & $158.2 \pm 51.2^{\mathrm{b}}$ \\
Data are mean $\pm \mathrm{SD} ; \mathrm{n}=5$; a $<<0.01$, vs Sham operated group; $\mathrm{b}<0.05, \mathrm{c}<<0.05$ vs OA model control group & \\
\hline
\end{tabular}

cartilage spontaneously releases PGE2 at 50-fold higher levels than in normal cartilage (Wang et al., 2010b). Also, NO has been identified as another agent in OA. NO is produced in large amounts by chondrocytes upon pro-inflammatory cytokine stimulation, and correlates with the pathophysiological changes in chondrocytes (Schwager et al., 2011). In addition, proinflammatory cytokines IL-1 $\beta$, IL- 6 and TNF- $\alpha$ have been suggested as key players in OA pathogenesis, and they are of an important role for development of synovitis and destruction of cartilage matrix (Bondeson et al., 2006; Goekoop et al., 2010).

The levels of PGE2, NO, TNF- $\alpha$, IL-6 and IL-1 $\beta$ in synovial fluids were markedly higher in OA group than in sham operated group $(\mathrm{p}<0.01)$. In SSG group, the PGE2 , NO, TNF- $\alpha$, IL-6 and IL-1 $\beta$ secretion reduced $13.9,18.9,22.7,38.5$ and $28.0 \%$ respectively com-pared to the OA group $(\mathrm{p}<0.05 ; \mathrm{p}<0.01)$ (Table II), suggesting SSG inhibited the synovial inflammation.

Increased MMP expression can be detected in the early stages of OA (Pelletier et al., 1990). MMP-3 can degrade the proteoglycan of cartilage (Yamanaka et al., 2000), while MMP-13 is known to degrade collagens and glycosaminoglycans (Roberts et al., 2000). As seen in Figure 3, SSG showed the beneficial effect on MMP-13 and MMP-3 mRNA and protein expression in cartilage which are related to cartilage matrix degradation $(\mathrm{p}<0.05)$.

\section{Discussion}

Alternative and complementary therapeutic approaches, such as the use of a wide array of herbal and nutritional manipulations, are becoming popular for relieving symptoms of OA (Pirotta, 2010). Notably, some components of soy have great potential in OA control. It has been demonstrated (Arjmandi et al., 2004) that $40 \mathrm{~g}$ of supplemental soy protein daily for 3 months improved OA-associated symptoms, such as range of motion and several factors associated with pain and quality of life, in comparison to same dosage of milk-based protein. Furthermore, biochemical markers of cartilage metabolism further supported the efficacy of soy protein supplementation in relieving symptoms of $\mathrm{OA}$ as indicated by a significant increase in serum level of insulin-like growth factor-I, a growth factor associated with cartilage synthesis, and a significant decrease in serum level of glycoprotein 39, a marker of cartilage degradation (Arjmandi et al., 2004). Besides, avocado/soybean unsaponifiables (ASU), a new antiarthritic agent derived from unsaponifiable residues of avocado and soybean oils mixed in the ratio of 1:2 respectively, has also been recommended for treat -ing OA with published basic and clinical trials in animals and humans (Maheu et al., 1998; Appelboom et al., 2001; Kut-Lasserre et al., 2001; Henrotin et al., 2003), thus considered a symptomatic disease-modifying osteo -arthritic compound (Lippiello et al., 2008). Metaanalysis data support better chances of success in patients with knee OA than in those with hip OA (Christensen et al., 2008). Therefore, components of soy 


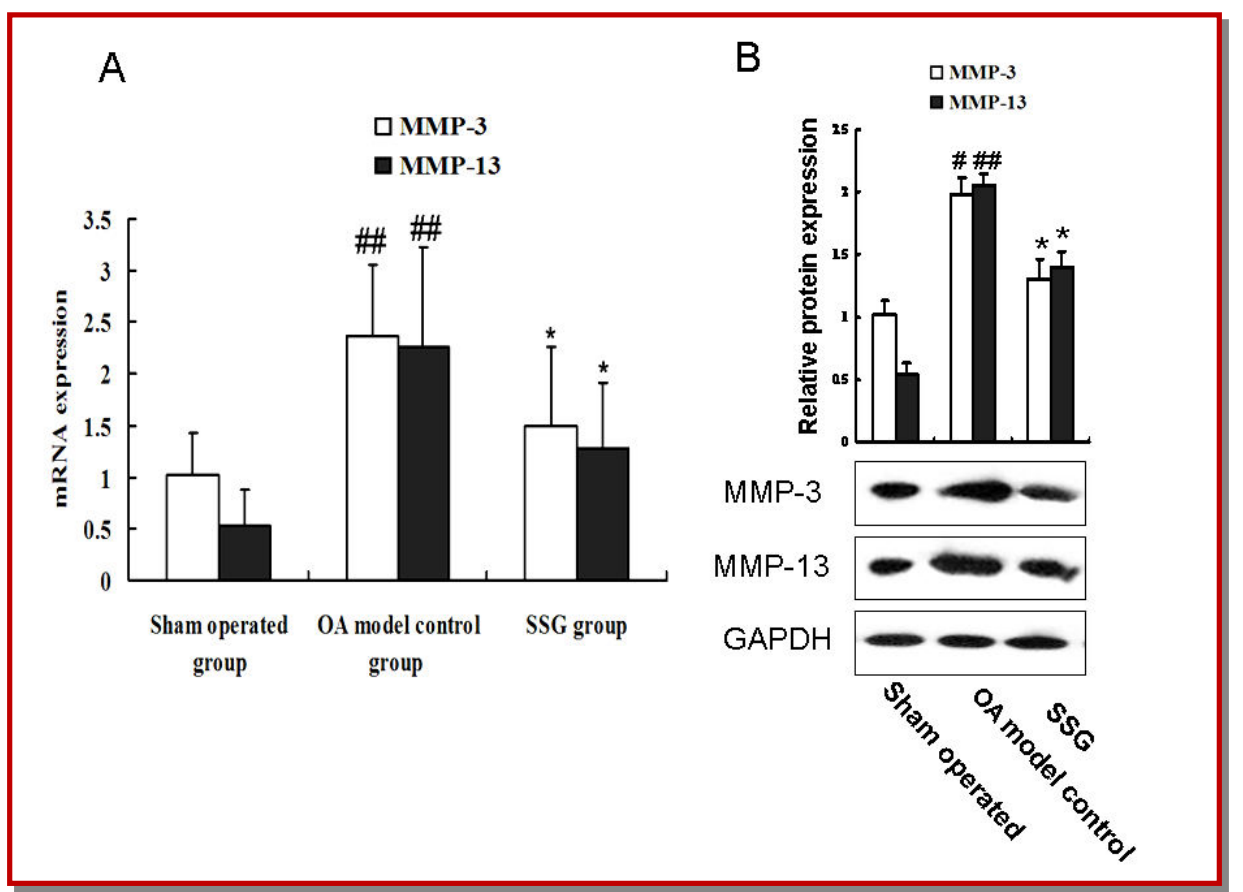

Figure 3: The mRNA and protein expression levels of MMP-3 and MMP-13 were determined by RT-PCR (A) and western blot (B) respectively

$\#$ \# $<<0.01$, vs Sham operated group; * $\mathrm{p}<0.05$, vs OA model control group

seem to be the feasible alternative to the control of OA.

Similar to the soy protein and ASU, SSG, a traditional Chinese medicine for "bi syndrome of bone" and "knee pain", also comes from the soy. SSG has been used for the treatment of joint pain and arthritis by Chinese medical doctor for over 2000 years. From the unique perspective of Chinese medicine, SSG has the effects of relieving exterior syndrome, activating blood circulation, detumescence and dehumidify. These effects, interpreted as analgesia, anti-inflammatory, promoting microcirculation and reducing swelling respectively in modern pharmacology, are all helpful for OA control. This study has shown that SSG as the feed material reduced the severity of the cartilage degeneration and delayed the degradation of cartilage matrix on rabbit knee OA model, indicating that SSG has a protective activity for OA.

Many researches have demonstrated that enzymatic cleavage by MMPs together with pro-inflammatory cytokines plays critical roles in the initiation and progression of cartilage destruction (Ju et al., 2010). The results of this study demonstrated that the contents of PGE2, NO, TNF- $\alpha$, IL-6 and IL-1 $\beta$ in synovia and expressions of MMP-3, MMP-13 in cartilage were elevated in OA model group, whereas the elevation of PGE2, NO, TNF- $\alpha$, IL-6, IL-1 $\beta$, MMP-3 and MMP-13 levels were significantly suppressed in SSG groups. Interestingly, these effects are similar to the antiosteoarthritic mechanism of ASU (Maheu et al., 1998; Appelboom et al., 2001; Kut-Lasserre et al., 2001;
Henrotin et al., 2003).

In addition, SSG is widely used in Chinese medicine, and also a kind of processed food - bean sprout, so its excellent safety record is reassuring. In the present study, no observable adverse effect of SSG as the feed material was seen during and after the 12-week feeding.

\section{References}

Appelboom J, Schuermans G, Verbruggen G, Henrotin Y, Reginster JY. Symptoms modifying effect of avocado/ soybean unsaponifiables (ASU) in knee osteoarthritis. Scand J Rheumatol. 2001; 30: 242-47.

Arjmandi BH, Khalil DA, Lucas EA, Smith BJ, Sinichi N, Hodges SB, Juma S, Munson ME, Payton ME, Tivis RD, Svanborg A. Soy protein may alleviate osteoarthritis symptoms. Phytomedicine 2004; 11: 567-75.

Bondeson J, Wainwright SD, Lauder S, Amos N, Hughes CE. The role of synovial macrophages and macrophage-produced cytokines in driving aggrecanases, matrix metalloproteinases, and other destructive and inflammatory responses in osteoarthritis. Arthritis Res Ther. 2006; 8: R187.

Cake MA, Read RA, Guillou B, Ghosh P. Modification of articular cartilage and subchondral bone pathology in an ovine meniscectomy model of osteoarthritis by avocado and soya unsaponifiables (ASU). Osteoarthritis Cartilage. 2000; 8: 404-11.

Cameron M, Gagnier JJ, Little CV, Parsons TJ, Blümle A, Chrubasik S. Evidence of effectiveness of herbal medicinal products in the treatment of arthritis. Part I: 
Osteoarthritis. Phytother Res. 2009; 23: 1497-515.

Christensen R, Bartels EM, Astrup A, Bliddal H. Symptomatic efficacy of avocado-soybean unsaponifiables (ASU) in osteoarthritis (OA) patients: A meta-analysis of randomized controlled trials. Osteoarthritis Cartilage. 2008; 16: 399408.

Echtermeyer F, Bertrand J, Dreier R, Meinecke I, Neugebauer K, Fuerst M, Lee YJ, Song YW, Herzog C, Theilmeier G, Pap T. Syndecan-4 regulates ADAMTS-5 activation and cartilage breakdown in osteoarthritis. Nat Med. 2009; 15: 1072-76.

Felson DT, Lawrence RC, Dieppe PA, Hirsch R, Helmick CG, Jordan JM, Kington RS, Lane NE, Nevitt MC, Zhang Y, Sowers M, McAlindon T, Spector TD, Poole AR, Yanovski SZ, Ateshian G, Sharma L, Buckwalter JA, Brandt KD, Fries JF. Osteoarthritis: New insights. Part 1: The disease and its risk factors. Ann Intern Med. 2000; 133: 635-46.

Getzy LL, Malemud CJ, Goldberg VM, Moskowitz RW. Factors influencing metachromtic staining in paraffin-embedded sections of rabbit and human cartilage: A comparison of the safranin $\mathrm{O}$ and toluidine blue techniques. J Histotechnol. 1982; 5: 111-16.

Goekoop RJ, Kloppenburg M, Kroon HM, Frölich M, Huizinga TW, Westendorp RG, Gussekloo J. Low innate production of interleukin-1beta and interleukin-6 is associated with the absence of osteoarthritis in old age. Osteoarthritis Cartilage. 2010; 18: 942-47.

Henrotin YE, Sanchez C, Deberg MA, Piccardi N, Guillou GB, Msika P, Reginster JY. Avocado/soybean unsaponifiables increase aggrecan synthesis and reduce catabolic and proinflammatory mediator production by human osteoarthritic chondrocytes. J Rheumatol. 2003; 30: 1825-34.

Hua B, O'Brien K. Osteoarthritis and Chinese Medicine: An overview of theories and evidence. J Chinese Med. 2010; 94: $1-14$.

Ju XD, Deng M, Ao YF, Yu CL, Wang JQ, Yu JK, Cui GQ, Hu YL. Protective effect of sinomenine on cartilage degradation and chondrocytes apoptosis. Yakugaku Zasshi. 2010; 130: 1053-60.

Kut-Lasserre C, Miller CC, Ejeil AL, Gogly B, Dridi M, Piccardi N, Guillou B, Pellat B, Godeau G. Effect of avocado and soybean unsaponifiables on gelatinase A (MMP-2), stromelysin 1 (MMP-3) and tissue inhibitors of matrix metalloproteinase (TIMP-1 and TIMP-2) secretion by human fibroblasts in culture. J Periodontol. 2001; 72: 1685-94.

Lippiello L, Nardo JV, Harlan R, Chiou T. Metabolic effects of avocado/soy unsaponifiables on articular chondro -cytes. Evid Based Complement Alternat Med. 2008; 5: 19197.
Maheu E, Mazières B, Valat JP, Loyau G, Le Loët X, Bourgeois $\mathrm{P}$, Grouin JM, Rozenberg S. Symptomatic efficacy of avocado/soybean unsaponifiables in the treatment of osteoarthritis of the knee and hip. Arthritis Rheum. 1998; 41: 81-91.

Pelletier JP, Jovanovic D, Fernandes JC, Manning P, Connor JR, Currie MG, Di Battista JA, Martel-Pelletier J. Reduced progression of experimental osteoarthritis in vivo by selective inhibition of inducible nitric oxide synthase. Arthritis Rheum. 1998; 41: 1275-86.

Pelletier JP, Mineau F, Faure MP, Martel-Pelletier J. Imbalance between the mehanisms of activation and inhibition of metallo-proteases in the early lesions of experimental osteoarthristis. Arthritis Rheum. 1990; 33: 1466-76.

Pirotta M. Arthritis disease- The use of complementary therapies. Aust Fam Physician. 2010; 39: 638-40.

Roberts S, Caterson B, Menage J, Evans EH, Jaffray DC, Eisenstein SM. Matrix metalloproteinases and aggrecanase: Their role in disorders of the human intervertebral disc. Spine 2000; 25: 3005-13.

Schwager J, Hoeller U, Wolfram S, Richard N. Rose hip and its constituent galactolipids confer cartilage protection by modulating cytokine, and chemokine expression. BMC Complement Altern Med. 2011; 11: 105.

Scott D, Kowalczyk A. Osteoarthritis of the knee. Am Fam Physician. 2008; 77: 1149-50.

Tramèr MR, Moore RA, Reynolds DJ, McQuay HJ. Quantitative estimation of rare adverse events which follow a biological progression: A new model applied to chronic NSAIDs use. Pain 2000; 85: 169-82.

Wang HM, Liu JN, Zhao Y. Progress on integrated Chinese and Western medicine in the treatment of osteoarthritis. Chin J Integr Med. 2010a; 16: 378-84.

Wang P, Zhu F, Lee NH, Konstantopoulos K. Shear-induced interleukin-6 synthesis in chondrocytes: Roles of $\mathrm{E}$ prostanoid (EP) 2 and EP3 in cAMP/protein kinase A- and PI3-K/ Akt-dependent NF-kappaB activation. J Biol Chem. 2010b; 285: 24793-804.

Yamanaka H, Matsuda Y, Tanaka M, Sendo W, Nakajima $\mathrm{H}$, Taniguchi A, Kamatani N. Serum matrix metalloproteinase 3 as a predictor of the degree of joint destruction during the six months after measurement, in patients with early rheumatoid arthritis. Arthritis Rheum. 2000; 43: 85258

Yoshioka M, Coutts RD, Amiel D, Hacker SA. Characterization of a model of osteoarthritis in the rabbit knee. Osteoarthritis Cartilage. 1996; 4: 87-98. 